\title{
Kinetics of formation and structural rebuilding crystalline films
}

\author{
I. G. Popova ${ }^{\dagger, 1}$, A. V. Blagin ${ }^{1}$, L. V. Blagina², V. I. Lebedev ${ }^{3}$ \\ †inna111109@rambler.ru \\ ${ }^{1}$ Don State Technical University, Rostov-on-Don, 344000, Russia \\ ${ }^{2}$ Institute of Technology Don State Technical University, Volgodonsk, 347386, Russia \\ ${ }^{3}$ North Caucasus Federal University, Stavropol, 355017, Russia
}

\begin{abstract}
The problem of fundamental analysis of "semiconductor film - matrix crystal" heterostructures is described. It is displayed that a universal model can be built on the basis of the quantum-statistical approach using the representations self-organization of ordered structures in systems far from spinodal isotherms and phase diagrams illustrating the conditions for the formation of films are calculated. Phase transitions of the first kind as the rearrangements of the "order-disorder" type are described within the framework of quantum theory using the Dicke model. The apparatus of two-period Green's functions is used. The thinfilm structure energy is described using the pseudopotential of the crystal field, which has elements of the substrate symmetry. The influence of the surface passivation degree on the nature and relief of structural changes is shown. Signs of the formation of dimeric and dipole elements of the film relief are discussed. It is shown that the processes of particles desorption depend on the temperature and the quantity of deposited particles with which the atoms of the matrix crystal, i. e. silicon, are bound. The kinetic processes of the formation of film structures and rearrangements of their structure are shown as local phase transformations caused by the adsorption of atoms with different concentrations of the adsorbed second phase. The threshold character of the surface structure rearrangement is confirmed. It is assigned that the order parameter, determined by the shear component of the activation threshold for structure reconstruction and the level populations, depends on inhomogeneities in the distribution of atoms at the heterointerface. Under the considered conditions, these inhomogeneities are stationary nonlinear - kink-like perturbations. The characteristics of disturbances are determined by the degree of difference between the crystal chemical parameters of the matrix and deposited materials. The transition to the formation of the films at the macrolevel is interpreted as a process of self-organization of a two-dimensional structure.
\end{abstract}

Keywords: nonequilibrium phase transitions, order-disorder rearrangements, crystal formation kinetics.

УДК: 538.915

\section{Кинетика формирования и структурной перестройки кристаллических пленок}

\author{
Попова И. Г. ${ }^{\dagger, 1}$, Благин А. В. ${ }^{1}$, Благина Л. В. ${ }^{2}$, Лебедев В. И. ${ }^{3}$ \\ ${ }^{1}$ Донской государственный технический университет, Ростов-на-Дону, 344000, Россия \\ ${ }^{2}$ Институт технологий Донского государственного технического университета, Волгодонск, 347386, Россия \\ ${ }^{3}$ Северо-Кавказский федеральный университет, Ставрополь, 355017, Россия
}

Описана проблема фундаментального анализа гетероструктур «полупроводниковая пленка - матричный кристалл». Показано, что универсальная модель может быть построена на основе квантово-статистического подхода с использованием представлений о самоорганизации упорядоченных структур в системах, далеких от равновесия. Рассчитаны спинодальные изотермы и фазовые диаграммы, иллюстрирующие условия формирования пленок. Фазовые переходы I рода как перестройки типа «порядок-беспорядок» описываются в рамках квантовой теории с использованием модели Дикке. Использован аппарат двухпериодных функций Грина. Энергия тонкопленочной структуры описана с применением псевдопотенциала поля кристалла, обладающего элементами симметрии подложки. Показано влияние степени пассивации поверхности на характер и рельеф структурных изменений. Обсуждаются признаки формирования димерных и дипольных элементов рельефа пленки. Показано, что процессы десорбции частиц зависят от температуры и от того, с каким количеством осаждаемых частиц связаны атомы матричного кристалла - кремния. Кинетические процессы образования пленочных структур и перестройки их структуры 
описаны как локальные фазовые превращения, обусловленные адсорбцией атомов с различными концентрациями адсорбирующейся второй фазы. Подтвержден пороговый характер перестройки поверхностной структуры. Установлено, что параметр порядка, определяемый сдвиговой компонентой порога активации рекострукции структуры и заселенностями уровней, зависит от неоднородностей в распределении атомов на гетерогранице. В рассмотренных условиях эти неоднородности представляют собой стационарные нелинейные - кинкоподобные возмущения. Характеристики возмущений определяются степенью различия кристаллохимических параметров матричного и осаждаемого материалов. Переход к образованию пленок на макроуровне интерпретирован как процесс самоорганизации двумерной структуры.

Ключевые слова: неравновесные фазовые переходы, перестройки «порядок-беспорядок», кинетика кристаллообразования.

\section{1. Введение}

Двумерные структуры, к которым условно можно отнести кристаллические пленки толщиной до $30 \ldots 50$ нм (верхние значения - при условии малости эффективной массы носителей [1]) в последние десятилетия стали одним из базовых компонентов электронной техники. Разработано большое количество методов их получения. Однако в ряде случаев стандартные технологии не позволяют получить материалы требуемого качества. В первую очередь это относится к структурам сложного состава и топологии [2]. Готовые модели формирования таких пленок, которые позволили бы отработать методику их получения с хорошей воспроизводимостью, отсутствуют. Возникает необходимость в ряде дорогостоящих экспериментальных исследований, позволяющих подобрать параметры технологии [3]. Таким образом, существует проблема фундаментального рассмотрения кинетических микропроцессов и разработки моделей фазовых переходов I рода. В настоящей работе предпринята попытка модельного описания кинетики роста тонких пленок и структурных превращений в них в рамках квантово-статистической теории, в частности, формирования кластеров и процессов спинодального распада кристаллизующейся фазы.

\section{2. Построение исходной модели эпитаксиального образования сложных пленок}

Поверхностную энергию кристаллической пленки будем описывать с использованием псевдопотенциала поля твердой фазы, несущего в себе элементы симметрии матричного кристалла [4]. Предполагаем, что рост пленки осуществляется в условиях эпитаксиального процесса из газовой или жидкой фазы. Неупорядоченная фаза равновесна с матричным кристаллом. Процесс осаждения начинается при охлаждении до температур ниже критической. Динамику зародышеобразования будем описывать гамильтонианианом

$$
\begin{aligned}
H & =\sum_{k}\left(\frac{k^{2}}{2 m}-\lambda\right) a_{k}^{+} a_{k}+ \\
& +\frac{1}{2 v_{k_{1}, k_{2}, k_{1}^{\prime}, k_{2}^{\prime}}} V\left(k_{1}-k_{1}^{\prime}\right) a_{k 1}^{+} a_{k 2}^{+} a_{k_{1}^{\prime}} a_{k_{2}^{\prime}}+\sum_{y, k} U_{G} a_{k j}^{+} a_{k j},
\end{aligned}
$$

(используется представление вторичного квантования, в выражении (1) $V\left(k_{1}-k_{1}^{\prime}\right)$ - потенциал парного взаимо- действия адатомов; $U_{G}-$ псевдопотенциал матричного поля, $j-$ номер узла, $a_{k}^{+}$и $a_{k}$ - операторы рождения и уничтожения частиц, для которых справедливы правила перестановок Бозе).

Концентрация осажденных частиц (конденсат) описывается методом двухпериодных функций Грина (ФГ), описанным нами в [4]. Их будем представлять в виде $G_{k k^{\prime}}\left(t_{1}, t_{2}\right)=\left\langle\left\langle a_{k}^{+}\left(t_{1}\right) a_{k^{\prime}}\left(t_{2}\right)\right\rangle\right\rangle ; \quad \Gamma_{k k^{\prime}}\left(t_{1}, t_{2}\right)=\left\langle\left\langle a_{k}^{+}\left(t_{1}\right) a_{k^{\prime}}^{+}\left(t_{2}\right)\right\rangle\right\rangle$. Нормальную и аномальную функции будем представлять Фурье-образами. Запишем уравнения для них с применением выражения (1):

$$
\begin{gathered}
G_{k k^{\prime}}(\omega)[\omega-\varepsilon]=1+\Delta \Gamma_{k k^{\prime}}(\omega), \\
\Gamma_{k k^{\prime}}(\omega)[\omega+\varepsilon]=\Delta^{+} G_{k k^{\prime}}(\omega), \\
\Delta^{+}=-\frac{1}{v_{k_{1}, k_{2}, k_{1}^{\prime}, k_{2}^{\prime}}} V\left(k_{1}-k_{1}^{\prime}\right)\left\langle a_{k 1}^{+}(1) a_{k 2}^{+}(1)\right\rangle .
\end{gathered}
$$

где $\varepsilon-$ энергия в расчете на один адатом, $\Delta-$ полоса энергий в спектре возбуждений монослоя, осажденного на матрице конденсата. Возникновение этой полосы является пороговым условием осаждения неупорядоченной фазы. Решение уравнений (2) и (3) дает

$$
G(\omega)=\frac{\omega+\varepsilon}{\omega^{2}-\mathrm{E}^{2}}, \quad \Gamma(\omega)=\frac{\Delta^{+}}{\omega^{2}-\mathrm{E}^{2}} .
$$

$\mathrm{E}(\omega)=\left(\varepsilon^{2}+|\Delta|^{2}\right)^{1 / 2}-$ энергия возбуждений в расчете на частицу. Из (4) и (5) с учетом Фурье-образов ФГ получаем интегральное уравнение, определяющее отмеченную выше полосу энергий частиц осаждаемого вещества:

$$
\Delta=-\sum V\left(k, k^{\prime}\right) \frac{\Delta}{\sqrt{\varepsilon^{2}+\left|\Delta^{2}\right|}} t h \frac{\sqrt{\varepsilon^{2}+\left|\Delta^{2}\right|}}{2 \Theta} .
$$

Поскольку пороговое условие осаждения $(\Delta \neq 0)$ известно, оказывается возможной запись уравнения спинодальной изотермы для кристаллизации сплошной пленки.

Помимо потенциала $V\left(k, k^{\prime}\right)$ попарного взаимодействия осаждаемых частиц, в энергии возбуждений $\varepsilon$ присутствует псевдопотенциал их взаимодействия с матричным кристаллом.

\section{3. Нахождение энергии активации и экспериментальная верификация}

Энергия одночастичных возбуждений $\varepsilon$ определяется кинетической энергией частиц и полем подложки $U$. При высокой температуре кинетическая энергия частиц 
пропорциональна температуре, поэтому можно положить её равной $f T$, где $f-$ константа, определяемая степенями свободы осаждаемых частиц.

Запишем потенциал матричного поля как $U(T)=U_{0} \exp (-e / k T)$.

Здесь $e-$ среднее значение этого потенциала на масштабах порядка постоянной кристаллической решетки; величина $U_{0}$ может быть найдена из условия нормировки. Уравнение (6) решалось нами в среде MathCAD. На Рис. 1 показано решение - спинодальная изотерма формирования сплошной пленки, где параметром выступает потенциал $V$ парного взаимодействия.

Это решение носит оценочный характер для определения локальных взаимодействий осаждаемых частиц (как между собой, так и с матричным кристаллом). Такие взаимодействия приводят к вариации критической температуры зародышеобразования. Полоса энергий $\Delta$ по сути, представляет собой активационный порог адсорбции. Выражение (6) позволяет оценить его зависимость от интенсивности взаимодействия - межчастичного на уровне осаждаемых атомов и с кристаллической решетки. Можно также качественно определить зависимость энергии активации от степени пассивации и температуры матричного кристалла.

Для экспериментальной верификации нами использовались данные [5] - работы, посвященной исследованию

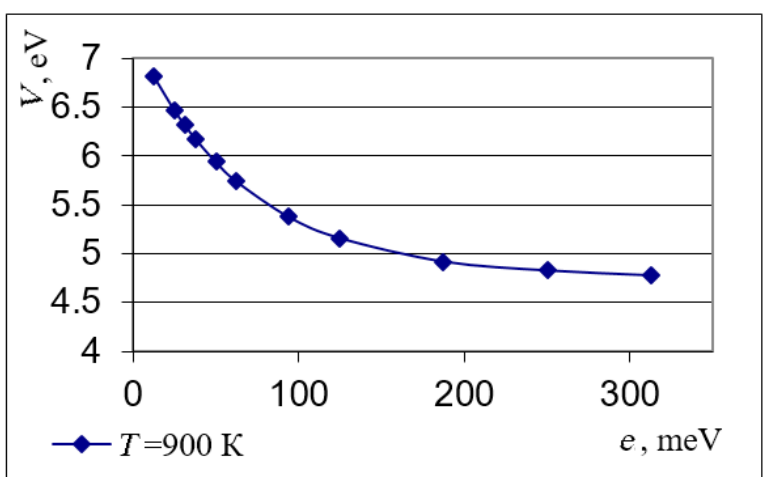

Рис. 1. Изотерма ( $T=900 \mathrm{~K})$ спинодального распада сплошной пленки.

Fig. 1. Isotherm $(T=900 \mathrm{~K})$ of spinodal decomposition of a continuous film. зародышеобразования двумерных структур - металлических пленок редких земель (Eu, Sm и $\mathrm{Yb}$ ), осажденных на поверхность кремния. Достаточно четкие закономерности зависимости активационного порога $E_{d}$ десорбции от степени пассивации $\Theta$, установленные авторами [5].

В системе $3 \times 2$ (Рис. 2 a) каждая из осажденных частиц связана с 2-мя атомами кремния, при этом если межчастичная дистанция вдоль ряда равна $2 a$, то между рядами дистанция составляет $3 a$. Здесь $a-$ постоянная решетки матричного кристалла кремния.

Условия эпитаксии резко изменяются: требуемое увеличение порога десорбции на 1.2 эВ не может быть достигнуто в силу слишком больших межчастичных расстояний. Авторы [5] делают предположение о перестройке поверхностных слоев Si. Например, возможно образование димеров и диполей из поверхностных атомов кремния, изолированных от осажденных атомов. Рост степени пассивации вызывает возникновение более плотной двумерной структуры $-5 \times 1$, что отображено на Рис. 2 b. В данной системе генерируются параллельно расположенные цепочки осажденных атомов. Если степень пассивации продолжает расти, возникает самая плотноупакованная система $2 \times 1$ (Рис. 2 с). В ней на поверхности нет «промежутков» между связями кремний-металл.

То есть, с ростом концентрации осаждаемых частиц число поверхностных атомов кремния, связанных только друг с другом, уменьшается. Соответственно, исчезают димеры и уменьшается порог десорбции $E_{d}$. Таким образом, потенциал попарного взаимодействия адатомов от степени пассивации не зависит, полный потенциал и вариации энергии активации обусловлены матричным полем кристалла. Если указанные величины меняются скачком, это может быть вызвано только перестройкой структуры пленки.

Рассмотрим далее зависимость $E_{d}$ от температуры. В качестве экспериментальных данных обратимся к данным работы [6], где рассматривается кинетика термодесорбции в паре кремний-тантал. Температурная зависимость в «обращенных» координатах $\left(\ln \left((d N / d t) / N, T^{-1}\right)\right.$ дает возможность определить тенденцию изменения углового коэффициента и, таким образом, оценить зависимость $E_{d}(T)$.

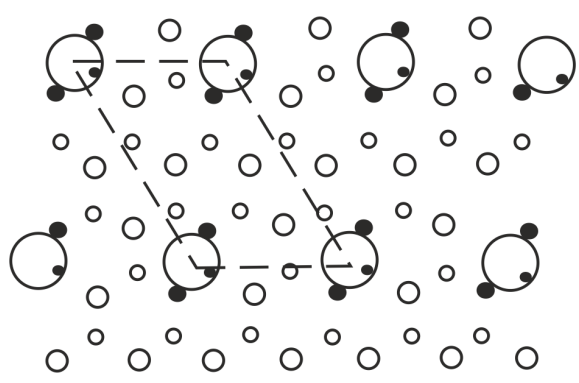

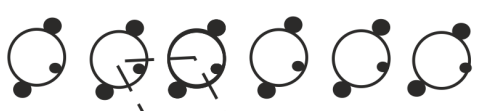
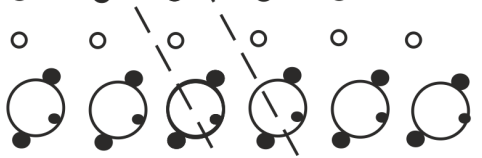

$0_{0}^{\circ} 0_{0}^{0}, 0,0,0.0$

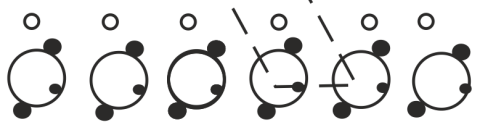

○

○

o

0

o

o
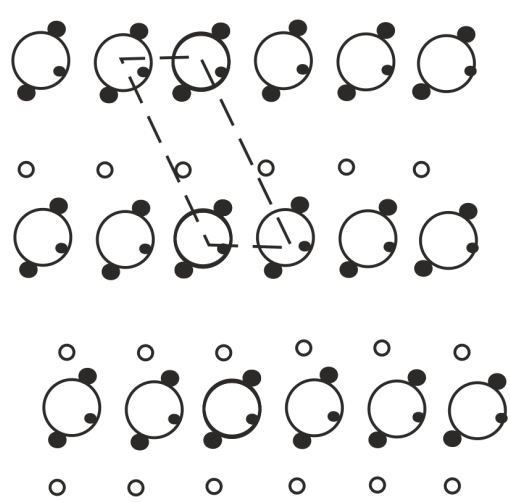

b

c

Рис. 2. Конфигурации осаждаемых на кремнии атомов: $3 \times 2$ (a); $5 \times 1$ (b); $2 \times 1$ (c) [5].

Fig. 2. Configurations of atoms deposited on silicon: $3 \times 2$ (a); $5 \times 1$ (b); $2 \times 1$ (c) [5]. 


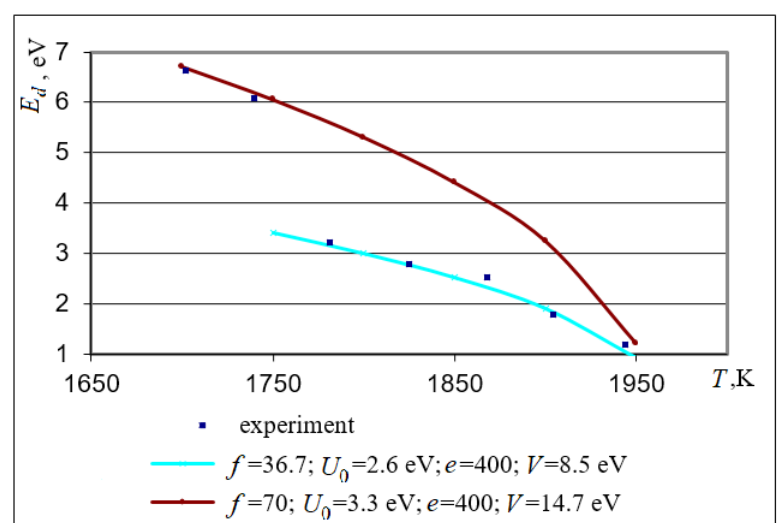

a

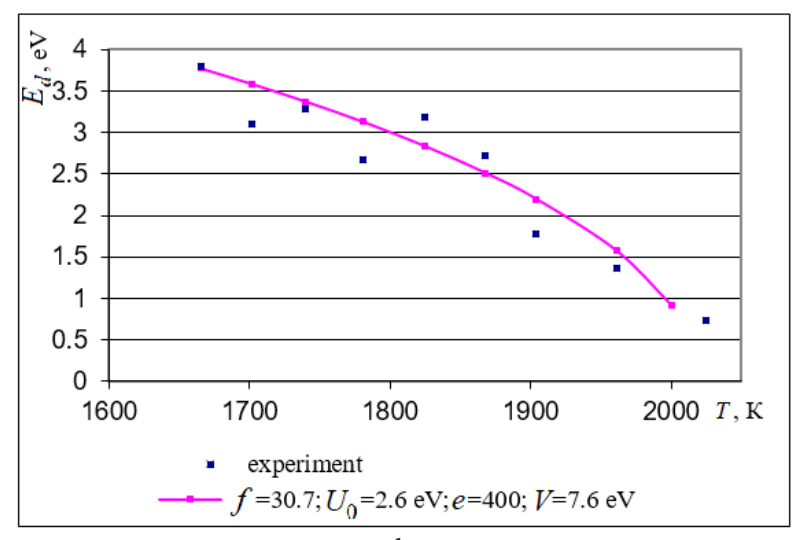

b

Рис. 3. (Color online) Экспериментальная зависимость $E_{d}(T)$, результаты [6] и теоретические зависимости для уровней пассивации $\Theta$ : 0.2 (a); 0.8 (b).

Fig. 3. (Color online) Experimental dependence $E_{d}(T)$, results [6] and theoretical dependences for passivation levels $\Theta: 0.2$ (a); 0.8 (b).

При уровне $\Theta=0.2$ зависимость энергии активации от температуры имеет монотонный характер и хорошо согласуется с нашей моделью для обоих случаев. Две ветви с различными значениями $E_{d}$ и в случае зависимости $E_{d}(T)$ свидетельствуют о перестройках в структуре осажденной пленки (Рис. 3a). При $\Theta=0.8$ прослеживается некоторый разброс, что по-видимому, вызвано неравномерностью потока при повышении уровня осаждения. Однако также можно говорить о соответствии расчетных и экспериментальных данных (Рис. 3 b).

Весьма характерно проявляются кинетические закономерности, связанные с пассивацией поверхности, в процессах взаимодействия карбида кремния, композитов ZnMnTe/ZnMgTe, кристаллизации графена на подложках соединений, обладающих сложными магнитными свойствами, например, MnO [7-12]. Так, гидрированная поверхность раздела SLG/H:MnO (здесь SLG - монослой графена) обладает существенно меньшей работой выхода носителей заряда по сравнению с системой без водорода. Нарушение стехиометрии по кислороду приводит к уменьшению энергии адсорбции [13]. Размерные эффекты в тонких пленках на основе систем А3В5 с числом компонентов 3 и более приводят в ряде случаев к модуляции поверхностной энергии [14,15].

\section{4. Перестройки двумерной структуры осаждаемой пленки}

Выше говорилось о нескольких свидетельствах перестройки структуры пленок. Очевидно, изменение интенсивности потока частиц является фактором, уводящим изначально равновесную двухфазную систему от стационарного развития. Это позволяет использовать представления о самоорганизации поверхностных процессов [4]. Современные аспекты базовых моделей роста приведены в монографии [16], а также в работах [17-19]. Техника моделирования кинетики осаждения подробно изложена в [20].

Однако и при описании перестройки поверхностных слоев весьма продуктивным является квантово-статистический подход. Он позволяет интерпретировать новые явления, которые часто рассматриваются в качестве эффектов развития дефектной структуры, как кинкоподобные стационарные возмущения. Рассмотрим процесс спонтанного осаждения частиц. Реконструкция поверхности обусловлена когерентным взаимодействием осаждаемых частиц, которое стимулировано неупорядоченным - спонтанным испусканием фононов. Возникают новые минимумы потенциала, в которые перемещаются частицы, и в силу периодичности матричного поля это приводит и к пространственным перемещениям адатомов, то есть, к структурной перестройке пленки. При этом перестройка, как и десорбция, также имеет пороговый характер: его определяет корреляция флуктуаций потенциала коллективной моды возмущений, в роли которой выступает акустическая ветвь фононов. Эти представления лежат в основе модели Дикке [4].

Гамильтониан в этой модели имеет две компоненты: бозонную и фермионную. Их взаимодействие описывается потенциалом $D(k q)$ :

$$
\begin{aligned}
H & =\sum h \omega_{k} b_{k}^{+} b_{k}+\sum \varepsilon_{k} a_{k}^{+} a_{k}+ \\
& +\sum D(k q)\left(b_{q} a_{k}^{+} a_{k-q}+a_{k} b_{k}^{+} b k_{K-q}\right),
\end{aligned}
$$

где операторы рождения и уничтожения возбуждений в системе осаждаемых частиц обозначены $b_{k}^{+} b_{k}$ и $a_{\mathrm{ak}}^{+} a_{\mathrm{\alpha} k}$. Энергия фононов $\hbar \omega_{k}$, активационный порог перестройки в расчете на одну частицу $\varepsilon_{k}$. Операторы с $a=1.2$ описывают фермионную компоненту, для бозонной используются числа заполнения $b_{k}^{+} b_{k}$.

Термодинамические следствия решений уравнений модели указывают на фазовые переходы II рода [4]. Сама структурная перестройка интерпретируется как акт дефектообразованиея, охватывающего поверхностные слои, причем, концентрация дефектов определяется температурой и отображается уравнением

$$
\begin{aligned}
& {\left[(\hbar \omega)^{4}+4 D^{-4} \xi^{2}\right]^{1 / 2}=} \\
& =D^{2} \operatorname{th}\left\{\left[(\hbar \omega)^{4}+4 D^{-4} \xi^{2}\right]^{1 / 2} / 2 \hbar \omega \Theta\right\}
\end{aligned}
$$

Здесь $\xi=\langle n\rangle / N-$ характеристический параметр, $\Theta_{\mathrm{C}}$ - критическая температура. 
Дальнейшее построение модели включает уравнения движения Гейзенберга, где вводится параметр порядка $\eta_{k}=\left\langle b_{k}^{+}\right\rangle$, определяемой сдвиговой компонентой активационного порога структурной перестройки, потенциалом поля деформаций $h_{k}=\left\langle d_{k}\right\rangle$ (которое сопряжено c упомянутой компонентой), а также средними заселённостями уровней $n_{k 1}$ и $n_{k 2}$. На их величины в значительной степени влияют релаксационные процессы, описываемые диссипативной функцией вида $\left(n_{k \alpha}-n_{k \alpha}^{e}\right) / \tau_{s}$. Получим безразмерные уравнения этих процессов, используя $\eta$ - параметр порядка, нормированный на функцию $\eta_{m}^{-2}=\tau_{h} \tau_{s} D^{2}$, сопряжённый потенциал $\mathrm{h}-$ (нормировка на функцию $\left.h_{m}^{-1}=\tau_{\eta}\left(\tau_{h} \tau_{s}\right)^{1 / 2} D^{2} / 2\right)$, и варьируемую величину $S$ (нормировка на функцию $S_{c}^{-1}=\tau_{\eta} \tau_{h} D^{2} / 4$ ):

$$
\begin{gathered}
\tau_{\eta} \dot{\eta}=-\eta+h, \\
\tau_{h} \dot{h}=-h+\eta S, \\
\tau_{h} \dot{S}=\left(S_{C}-S\right)+\eta h .
\end{gathered}
$$

Переход двухфазной системы в равновесие задается отрицательной обратной связью параметра порядка и сопряжённого потенциала с характеристикой $S$. Последняя модель была разработана Лоренцем и содержит параметр порядка $h$, сопряженный с ним потенциал $h$ и варьируемую характеристику $S$, имеющую смысл «управляющей» функции. Эта «классическая» синергетическая модель является практически универсальной для описания структурных фазовых переходов, трактуемых как процессы самоорганизации в системах, далеких от равновесия [2]. Плотность распределения частиц по состояниям $\langle n(t)\rangle$ определяется из известного уравнения Рэлера-Эберли [2]:

$$
\frac{\partial n}{\partial t}=-\frac{1}{\tau_{0}}\left\{\frac{N}{2}+n+\mu\left(\frac{N^{2}}{4}-n^{2}\right)\right\},
$$

На малых промежутках времен его решение может быть аппроксимировано выражением

$$
n(t)=\frac{1}{2 \mu}-\eta t h\left(\mu \eta \frac{t-t_{D}}{\tau_{0}}\right),
$$

Такие периодические отклонения в распределении частиц на поверхности раздела матричный кристалл - пленка относятся к нелинейным возмущениям типа кинков.

Величина $t_{D}$ имеет смысл времени задержки формирования указанных периодических конфигураций, по сути - задержки формирования импульса фононов и стимулируемых скачков энергий частиц. Значение этой величины приблизительно на порядок больше времени генерации самого импульса фонона. Наличие в функции плотности распределения частиц слагаемого, пропорционального $\langle n\rangle^{2}$ свидетельствует о когерентном характере возмущений на временах $t \sim t_{D}$. Эти возмущения, интерпретируемые как фононы «мягкой» моды, являются определяющими в процессах самоорганизации пленок и структурных перестроек в них.

\section{5. Заключение}

В работе описаны принципы моделирования процессов формирования тонких кристаллических пленок в рамках квантово-статистической теории (микроскопический подход) и синергетики (макроскопический подход). На основе гамильтониана Дикке построена модель первичного осаждения пленок и осуществлено моделирование начальной стадии кристаллообразования. Показано, что параметр порядка связан с наследованием пленкой параметров матричного кристалла, неоднородности в распределении атомов на поверхности раздела носят периодический характер в виде нелинейных стационарных возмущений - кинков, что обусловлено различием кинетических характеристик матричного и осаждаемого материалов. Проведено сравнение рассчитанных зависимостей активационного порога десорбции от температуры и степени пассивации поверхности матричного кристалла с экспериментальными данными других авторов, получено удовлетворительное соответствие результатов. Такое сравнение позволило объяснить особенности указанных зависимостей, что дает основания для разработки технологии воспроизводимого получения металлических пленок, рассматриваемых в качестве активных элементов электронных устройств.

\section{Литература/References}

1. C. Weisbuch, B. Vinter. Quantum semiconductor structures. Fundamentals and Applications. 611 (38), 217 (1991).

2. A. V. Blagin, V.V. Kalinchuk, V.I. Lebedev, L.S. Lunin. Physics of crystallization and defects of solid structures on micro- and nanolevels. Rostov-on-Don. SSC RAS (2009) 286 p. (in Russian) [А. В. Благин, В.В.Калинчук, B.И. Лебедев, Л.С. Лунин. Физика кристаллизации и дефектов твердотельных структур на микрои наноуровне. Ростов-на-Дону, ЮНЦ РАН (2009) 286 c.]

3. D. Bimberg, I.P. Ipatova, P.S. Kopyev, N. N. Ledentsov, V.G. Malyshkin, V.A. Shchukin. Phys. Usp. 167 (5), 552 (1997). (in Russian) [Д. Бимберг, И.П. Ипатова, П.С. Копьев, Н.Н. Леденцов, В.Г. Малышкин, В. А. Щукин. УФН. 167 (5), 552 (1997).] Crossref

4. V.I. Lebedev. Physics of phase transitions in defective and small-size crystals. Stavropol, SevKavGTU (2008) 227 p. (in Russian) [В.И. Лебедев. Физика фазовых переходов в дефектных и малоразмерных кристаллах. Ставрополь, СевКавГТУ (2008) 227 с.]

5. T.V. Krachino, M.V. Kuzmin, M.V. Loginov, M. A. Mittsev. Phys. Solid State. 40 (2), 371 (1998). (in Russian) [Т. В. Крачино, М. В. Кузьмин, М.В. Логинов, М.А. Митцев. ФТТ. 40 (2), 371 (1998).] Crossref

6. V.N. Ageev, E.Yu. Afanasyeva, N.D. Potekhina, A. Yu. Potekhin. Phys. Solid State. 42 (2), 347 (2000). Crossref

7. M.V. Gomoyunova, G.S. Grebenyuk, V.Yu. Davydov, I. A. Ermakov, I. A. Eliseev, A. A. Lebedev, S. P. Lebedev, E. Yu. Lobanova, A. N. Smirnov, D. A. Smirnov, I. I. Pronin. Phys. Solid State. 60 (7), 1423 (2018). (in Russian) [М. В. Гомоюнова, Г.С. Гребенюк, В.Ю. Давыдов, И.А. Ермаков, И.А. Елисеев, А.А. Лебедев, С.П. Лебедев, Е.Ю. Лобанова, А.Н. Смирнов, 
Д. А. Смирнов, И. И. Пронин. ФТТ. 60 (7),1423 (2018).] Crossref

8. I. V. Shtrom, V.F. Agekyan, A. Yu. Serov, N. G. Filosofov, R.R. Akhmadullin, D.E. Krizhkov, G. Karczewski. Semiconductors. 52 (4), 481 (2018). Crossref

9. V.V. Ilyasov, I. V. Ershov, I.G. Popova, K.D. Pham, Ch. V. Nguyen. Superlattices and Microstructures. 117, 72 (2018). Crossref

10. V. V. Ilyasov, I. G. Popova, I. V. Ershov. Applied Surface Science. 419, 924 (2017). Crossref

11. V.V. Ilyasov, I.G. Popova, I. V. Ershov, N.D. Chien, N. N. Hieu, Ch. V. Nguyen. Diamond \& Related Materials. 74, 31 (2017). Crossref

12. V. V. Ilyasov, B. Ch. Meshi, I. G. Popova, Ch. V. Nguyen, I. V. Ershov, N. D. Chien. Springer Proceedings in Physics. 175, 279 (2016). $\underline{\text { Crossref }}$

13. V.V. Ilyasov, O. M. Holodova, I. G. Popova, I.P. Gritsay, I. V. Ershov. Applied Surface Science. 462, 772 (2018). Crossref

14. A. V. Blagin, V.V. Nefedov, N.A. Nefedova. IOP Conf. Ser.: Earth Environ. Sci. 87 (9), 092005 (2017). Crossref
15. A. V. Blagin, V. V. Nefedov, N. A. Nefedova. Proceedings of the International Conference "Actual Issues of Mechanical Engineering" (AIME 2018). Advances in Engineering Research. Atlantis Press. 157, 95 (2018). $\underline{\text { Crossref }}$

16. R. Manijeh. Fundamentals of Solid State Engineering. 3rd ed. Springer (2009) 758 p. Crossref

17. E. Neyts, A. Bogaerts, M.C.M. van de Sanden. High Temperature Material Processes. 13 (3-4), 399 (2009). Crossref

18. A. V. Osipov. Phys.Chem.Mech.Surfaces. 8, 34 (1991). (in Russian) [А. В. Осипов. Поверхность. Физика, химия, механика. 8, 34 (1991).]

19. G. V. Dubrovsky, V. V. Kozachek. Colloid Journal. 56 (3), 356 (1994). (in Russian) [Г. В. Дубровский, В. В. Козачек. Коллоидный журнал. 56 (3), 356 (1994).]

20. S. V. Kolesnikov, A.M. Saletsky, S.A. Dokukin, A. L. Klavsyuk Mat. Modeling. 30 (2), 48 (2018). (in Russian) [C.В. Колесников, А.М. Салецкий, С. А. Докукин, А. Л. Клавсюк. Матем. Моделирование. 30 (2), 48 (2018).] 